\title{
Controle Eletrônico de Ganho Ultra-Rápido baseado em DSP aplicado a EDFAs em Redes Ópticas WDM
}

\author{
Juliano R. Fernandes de Oliveira, Júlio C. R. Fernandes de Oliveira, Adolfo F. Herbster e Elnatan C. Ferreira
}

\begin{abstract}
Resumo - Uma estrutura de controle eletrônico de ganho aplicado a EDFAs (Erbium Doped Fiber Amplifiers), baseada em DSP (Digital Signal Processor), é demonstrada experimentalmente, com ciclo de leitura, cálculo e atuação de 9us, habilitando a supressão de transiente do EDFA cuja dinâmica é de $150 \mu \mathrm{s}$, quando saturado. A supressão de transiente é analisada, alcançando um tempo de resposta de 150 $\mu$ s com overshoot/undershoot de $1,02 \mathrm{~dB}$ (add/drop de sete canais), habilitando o uso desta técnica para EDFAs em redes ópticas reconfiguráveis. Os resultados obtidos posicionam a técnica desenvolvida no estado da arte em termos de controle de ganho de EDFAs.
\end{abstract}

Palavras-chaves - Amplificadores ópticos, Redes reconfiguráveis, Processamento digital de sinais.

\begin{abstract}
An automatic electronic gain control structure applied to EDFAs (Erbium Doped Fiber Amplifiers) and based on DSP (Digital Signal Processor) is experimentally demonstrated, with a read, calculate and actuating cycle of $9 \mu \mathrm{s}$, enabling the EDFA transient suppression at $150 \mu$ s of the dynamic settling time, when saturated. The transient suppression (for seven-add/drop channels) is analyzed, reaching a maximum settling time of $150 \mu \mathrm{s}$, with maximum overshoot/undershoot of $1,02 \mathrm{~dB}$, enabling the use of this technique to EDFAs in reconfigurable optical networks. The obtained results align this developed technique on the state of the art in EDFAs controlled gain terms.
\end{abstract}

Keywords - Optical amplifiers, Reconfigurable networks, Digital signal Processing.

\section{INTRODUÇÃO}

O amplificador a fibra dopada com érbio (EDFA) proporciona amplificação de múltiplos sinais, independente de taxa de transmissão ou formato de modulação, habilitando a formação e evolução de redes ópticas multiplexadas em comprimento de onda (WDM) [1], representando um dos principais componentes destas redes [2]. Com o advento da nova geração de sistemas ópticos WDM que utilizam ROADMs (Reconfigurable Optical Add-Drop Multiplexers) de grau $\mathrm{N}$ ou switches ópticos capazes de prover reconfiguração entre os enlaces, o número de canais em cada enlace se torna uma variável aleatória. Conseqüentemente, os EDFAs, que possuem o ganho dependente do sinal de entrada, necessitam se adequar aos novos requisitos advindos

Juliano R. Fernandes de Oliveira, Júlio C. R. Fernandes de Oliveira e Adolfo F. Herbster, Fundação CPqD, Campinas, São Paulo, \{jrfo, julioc, adolfo\}@cpqd.com.br.

Elanatan C. Ferreira, DEMIC - Departamento de Eletrônica e Microeletrônica, FEEC - Faculdade de Engenharia Elétrica e Computação, UNICAMP - Universidade de Campinas, Campinas, São Paulo, elnatan@fee.unicamp.br. da reconfiguração, com o intuito de prover ganho constante independente do número de canais em sua entrada. Bruscas variações de potência de entrada, como as impostas pelas redes WDM reconfiguráveis, impossibilitam a supressão de transientes quando utilizadas as técnicas de controle de ganho desenvolvidas para redes WDM ponto-a-ponto. Devido a uma necessidade lenta de reconfiguração, os sistemas pontoa-ponto apresentam lentos mecanismos de controle projetados para lentas variações de potência induzidas pela degradação do enlace ou pela inserção manual e programada de canais. A ausência deste rápido controle transiente afeta os canais sobreviventes ao longo do transiente de controle, usualmente da ordem de milisegundos, causando penalidade devido ao excesso ou ausência de potência (remoção ou adição). Degradação sistêmica (BER) da rede devido ao baixo nível de sinal, e a danificação dos fotodetectores devido ao excesso de potência nos transmissores durante o transiente de potência representam significativas fontes de penalização nas redes ópticas reconfiguráveis [1].

Para solução deste fenômeno indesejado, vários esquemas de controle automático de ganho (AGC) eletrônico foram desenvolvidos [3]-[8]. Inicialmente, [3] e [4] propuseram um esquema de controle de ganho baseado em um laser em regime contínuo, utilizado apenas como um sinal para controle de saturação do EDFA. No entanto esta técnica além de obter resultados limitados, o canal extra, ocupa o espaço de um possível canal de dados, tornando este um esquema dispendioso de controle de ganho. Grande parte dos métodos de controle eletrônico encontrados na literatura realiza o controle de ganho através do ajuste da potência do laser de bombeio do EDFA. Os controles realimentados, com alimentação adiante ou ambos [5]-[8], são os mais utilizados e que apresentam resultados mais satisfatórios. No entanto, estes controladores foram desenvolvidos para redes fixas, não levando em consideração a existência do transiente de potência. Dessa maneira, o controlador garante ganho constante apenas em regime permanente, o que inviabiliza o uso destes controles de ganho para EDFAs em redes WDM reconfiguráveis.

Neste trabalho foi desenvolvido e validado experimentalmente um método ultra-rápido de controle automático de ganho para EDFAs baseado em um DSP, habilitando a supressão de transientes, e, conseqüentemente, garantindo a aplicabilidade dos EDFAs em redes WDM reconfiguráveis. Foram realizadas as etapas de projeto do hardware óptico, do hardware eletrônico, e a implementação do algoritmo do controlador PI embarcado no DSP para controle do nível do laser de bombeio. Apresentamos uma avaliação experimental do EDFA em torno da supressão de transientes e do tempo de controle, comparando-os com as 
especificações necessárias às redes WDM reconfiguráveis [9]-[10], demonstrando o êxito dos resultados obtidos.

\section{AMPLIFICADORES A FIBRA DOPADA COM ÉRBIO PARA REDES WDM RECONFIGURÁVEIS DE NOVA GERAÇÃO}

Os elementos básicos que constituem um amplificador óptico a fibra dopada com érbio são; a fibra dopada com érbio, o laser de bombeio, o acoplador WDM e os isoladores, como ilustrado na Fig. 1.

Com o aumento da potência na entrada do EDFA ocorre uma redistribuição da amplificação provida a todos os canais, resultando em uma diminuição de ganho por canal, podendo causar a perda do ponto de recepção do sinal (aumento da BER). Com a diminuição de potência na entrada do EDFA ocorre uma redistribuição da amplificação provida aos canais sobreviventes, que por sua vez, implica em excesso de amplificação por canal, podendo causar a saturação do fotodetector de recepção (danificando o fotodetector).

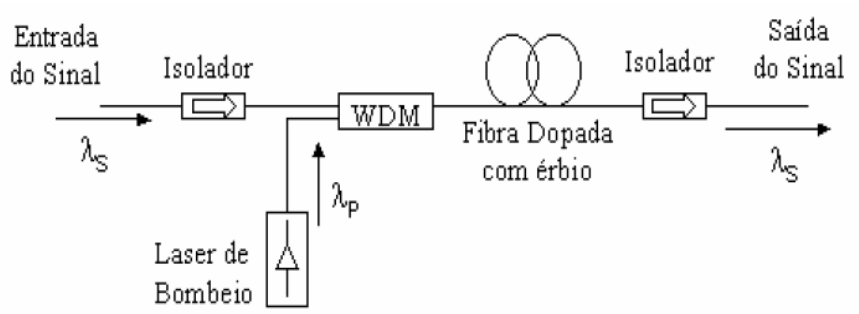

Fig. 1 - Configuração básica de um EDFA.

Trabalhos anteriores [5]-[8] demonstram uma maior eficiência dos controles eletrônicos que fazem uso da variação do bombeio para correção do ganho nos EDFAs. Porém, estes controles possuem um lento mecanismo de controle (da ordem de milisegundos), devido ao fato de serem projetados para redes ópticas fixas. Para redes WDM reconfiguráveis, requisitos exigentes devem ser obedecidos para uma adequada operação, entre eles destacam-se (considerando uma inserção/remoção de 7 canais) [10,11]:

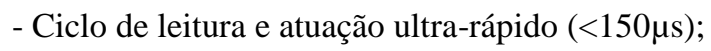

- Overshoots/Undershoots inferiores a $1 \mathrm{~dB}$;

- Tempo de estabilização inferior a $150 \mu$ s;

Estes severos requisitos, se atendidos pelo controle de ganho, garantem um impacto mínimo à rede óptica reconfigurável, minimizando os efeitos da inserção e remoção de canais de forma a habilitar a operação da rede óptica baseada em EDFAs.

Para obtenção de um controle de ganho de alta velocidade, neste trabalho, foram utilizados componentes eletrônicos de alta capacidade de processamento e baixo tempo de resposta para o controle da corrente do laser de bombeio, este realizado via um controlador PI embarcado no DSP.

\section{EDFA COM CONTROLE DE GANHO ELETRÔNICO VIA DSP}

Nesta seção descrevemos o desenvolvimento do EDFA separado nos seguintes tópicos: circuito óptico e eletrônico, análise sistêmica e, por fim, a caracterização temporal do EDFA com o AGC desenvolvido que é apresentada na seção IV.

No circuito óptico ilustrado na Fig. 2, em busca de equalização da banda e um melhor compromisso entre figura de ruído e potência de saída foi utilizada a configuração com bombeio bi-direcional, sendo o bombeio \#1 um laser de 980 $\mathrm{nm}$ e o bombeio \#2 um laser de $1480 \mathrm{~nm}$. A utilização de múltiplos estágios separados por um isolador óptico tem a função de bloquear a propagação da ASE retro-espalhada proveniente do segundo estágio, evitando, assim, a redução do ganho no primeiro estágio devido à diminuição de potência de ASE retroespalhada. Além dos parâmetros tradicionais, potência de saída, ganho e figura de ruído, neste EDFA o comprimento da fibra foi definido levando também em consideração a planicidade da banda $\mathrm{C}$ na faixa de potência de entrada de operação do EDFA.

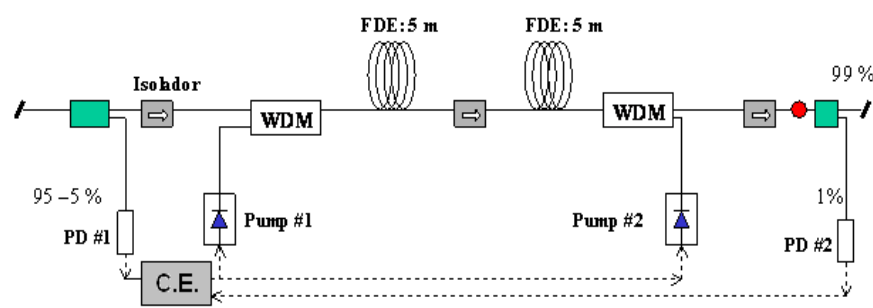

Fig. 2 - Configuração do circuito óptico do EDFA para redes WDM reconfiguráveis de nova geração.

O bloco do circuito eletrônico ilustrado na Fig. 2 (C.E.) é ilustrado detalhadamente na Fig. 3. Neste projeto se faz necessária a obtenção de um tempo de atuação associado ao ciclo de controle (leitura, cálculo de ganho e atuação) muito inferior à dinâmica do EDFA $(150 \mu \mathrm{s})$ para obter-se a supressão de transiente. Para isto, fez-se necessária à utilização de um núcleo de processamento baseado em um processador digital de sinais (DSP) capaz de prover um alto desempenho no processamento das operações matemáticas. No DSP, uma aritmética de ponto fixo é utilizada no processamento do controle de ganho de um controlador proporcional integral (PI) (sintonizado através do método da malha fechada de Ziegler-Nichols) utilizando as leituras da potência total da entrada e saída para determinar as constantes de controle do controlador PI que controla os dois lasers de bombeio do EDFA. Para medição das potências foi utilizado um amplificador logarítmico que provê uma faixa de leitura de potência da ordem de $60 \mathrm{~dB}$ óptico. Antes da passagem do valor das potências de entrada e saída para o conversor analógico digital, realizamos uma filtragem passiva com o objetivo da redução de ruídos de alta freqüência na potência a ser medida.

Para obtenção das métricas de tempo de controle e para permitir a supressão de transiente no EDFA foram utilizados conversores analógicos digitais (A/D) com 12 bits de resolução e taxa de amostragem de 1 MSPS (milhões de 
amostras por segundo). Conversores digitais analógicos (D/A) com 12 bits de resolução e taxa de amostragem de 2,47 MSPS também foram utilizados. Ambos (A/D e D/A) posuem interface de comunicação SPI (interface periférica serial), o que permitiu um tempo total de aquisição de aproximadamente $1,5 \mu \mathrm{s}(\mathrm{A} / \mathrm{D})$ e $1 \mu \mathrm{s}(\mathrm{D} / \mathrm{A})$.

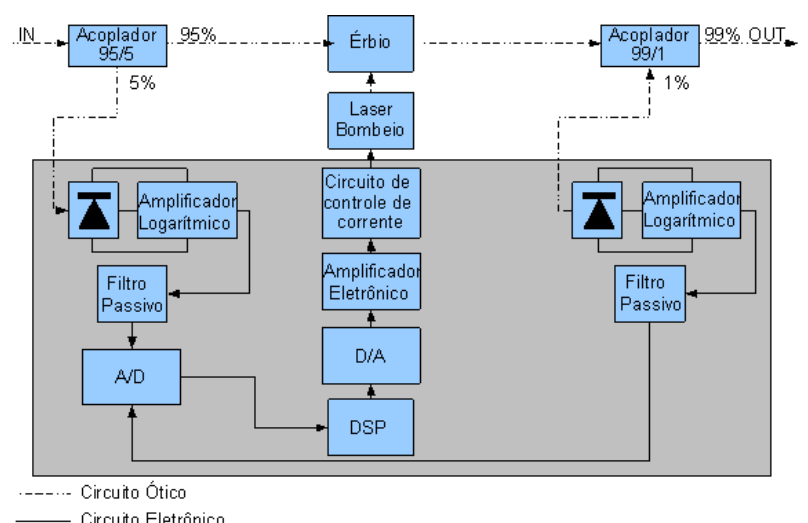

Fig. 3 - Circuito Eletrônico para controle da corrente do laser de bombeio de cada laser de bombeio do EDFA.

A taxa com a qual o ganho do EDFA varia de acordo com a potência do sinal de entrada, ou de bombeio, durante o período transiente, é determinada pelo tempo de vida do nível metaestável e pela potência de saída saturada [12]. As constantes de tempo da saturação do ganho e da recuperação do ganho, para os EDFAs, são da ordem de várias centenas de microssegundos [13]. EDFAs para redes ópticas WDM possuem, caracteristicamente, altos níveis de potência de saída, o que reduz essas constantes de tempo para a ordem de poucas centenas de microssegundos [14].

Foi obtido através do hardware eletrônico desenvolvido neste trabalho uma iteração do controle de ganho (aquisição de medidas, cálculos do controlador e atuação nos lasers de bombeio) com um tempo de aproximadamente $9 \mu$ s. Como o EDFA projetado possui tempo de vida do nível metaestável de aproximadamente $150 \mu$ s (tempo mínimo no qual o EDFA é capaz de transferir a variação da entrada para a saída), verificou-se que o controle de ganho deverá ocorrer em no máximo 16 iterações, para que seja atendida a condição plena de supressão de transiente, onde a correção do ganho é realizada em um tempo inferior à resposta do EDFA.

\section{TESTES SISTÊMICOS E CARACTERIZAÇÃO TEMPORAL}

O arranjo experimental utilizado para avaliar sistemicamente o desempenho do EDFA com a técnica de controle ultra-rápida desenvolvida é ilustrado pela Fig. 4.

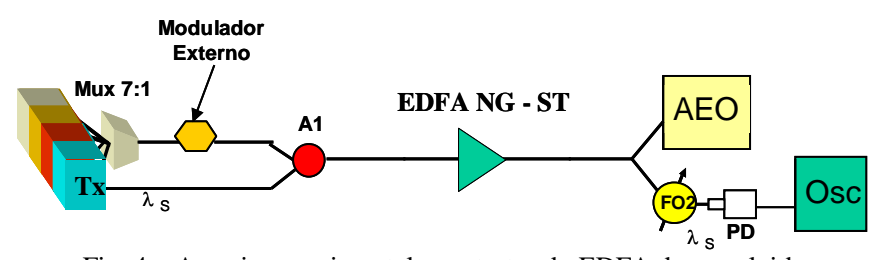

Fig. 4 - Arranjo experimental para testes do EDFA desenvolvido.
No arranjo experimental (Fig.4) são utilizados sete canais posicionados na banda $\mathrm{C}$ de acordo com a norma ITU G.694.2 (1531,12 nm; 1532,68 nm; 1535,04 nm; 1536,61 nm; $1538,98 \mathrm{~nm} ; 1540,56 \mathrm{~nm}$ e 1542,94 nm). Depois de equalizados estes canais são inseridos em um multiplexador de canais (Mux 7:1). Os canais multiplexados são modulados por uma chave acústico-óptica através de uma forma de onda quadrada de $100 \mathrm{~Hz}$ de freqüência (add/drop com intervalos de $5 \mathrm{~ms}$ ). Posteriormente, estes canais são inseridos em uma das entradas do splitter (A1), que em sua outra entrada possui a presença do canal sobrevivente $(1550,92 \mathrm{~nm})$. O canal sobrevivente não é modulado na análise do transiente de potência e do tempo de controle, sendo modulado a $10 \mathrm{~Gb} / \mathrm{s}$ para realização da análise sistêmica (diagrama de olho). Através de um gerador de onda acoplada a uma chave óptica, são simuladas 100 inserções/remoções por segundo para carregamentos de 7, 3 e 1 canais em um sistema WDM, ocasionando uma variação na potência de entrada de 9,6 e 3 $\mathrm{dB}$, respectivamente. Através deste comportamento, procurase emular o cenário de uma rede WDM reconfigurável baseada em ROADMs ou Cross-Connects. Neste cenário, o analisador de espectro óptico (OSA) é utilizado para análise dos canais na saída do EDFA, sendo, a resposta temporal do canal sobrevivente analisada através da passagem do sinal de saída por um filtro sintonizado em seu comprimento de onda, seguido por um fotodetector e um osciloscópio, para visualização dos transientes de potência do canal sobrevivente. Quanto à análise sistêmica, esta foi realizada qualitativamente por meio do uso de um osciloscópio de alta velocidade, obtendo o diagrama de olho frente às variações de potência na entrada do EDFA.

Para análise do EDFA com a técnica de controle desenvolvida, foram realizadas as seguintes análises:

i) Supressão de transientes de potência óptica no canal sobrevivente;

ii) Tempo de resposta de controle de ganho do canal sobrevivente (settling time).

iii) Desempenho sistêmico via de diagrama de olho;

Os resultados apresentados foram conduzidos para os ganhos de $20 \mathrm{~dB}$ e $18 \mathrm{~dB}$, sob as condições de adição/remoção de 3, 6 e $9 \mathrm{~dB}$ de potência óptica da entrada do $\operatorname{EDFA}(1 ; 3$ e 7 canais, respectivamente).

\section{A. Supressão de Transientes e Tempo de Resposta}

Para avaliação de desempenho do transiente de potência e do settling time para o canal sobrevivente o EDFA com o controle de ganho desenvolvido foi utilizado o arranjo experimental ilustrado pela Fig. 4. A validação do desempenho ao longo dos transientes se baseia na análise da potência de saída do canal sobrevivente do EDFA em função do tempo. A seguir, as Figs. 5 a 9, ilustram os dados obtidos na análise do comportamento transiente e do settling time.

A Fig. 5 ilustra a potência de saída do canal sobrevivente no EDFA sem controle de ganho para as situações de 
adição/remoção de 1 canal , 3 canais e 7 canais, equalizados em nível de potência (-20 dBm por canal) na entrada do EDFA. Para situação de adição/remoção de 1 canal $(3 \mathrm{~dB})$ na entrada (potência de entrada de $-17 \mathrm{dBm}$ com dois canais ou $-20 \mathrm{dBm}$ somente com o canal sobrevivente) verifica-se através da Fig. 5 que, para o EDFA sem controle de ganho, a saída do canal sobrevivente sofre uma variação de aproximadamente 1,6 dB. Esta variação da potência de saída do canal sobrevivente não corresponde aos $3 \mathrm{~dB}$ da variação da entrada devido o EDFA não o mesmo nível de saturação para ambos os níveis de potência de entrada aplicados. Para situação de adição/remoção de 3 canais na entrada do EDFA ( $6 \mathrm{~dB}$ de variação na potência de entrada, que vai de $-14 \mathrm{dBm}$ com quatro canais a $-20 \mathrm{dBm}$ somente com o canal sobrevivente), e 7 canais ( $9 \mathrm{~dB}$ de variação na potência de entrada, que vai de $-11 \mathrm{dBm}$ para oito canais a $-20 \mathrm{dBm}$ somente com o canal sobrevivente) são verificadas, através da Fig. 5, variações da potência de saída do canal sobrevivente de aproximadamente 3,4 dB e 5,9 dB respectivamente. Essas variações bruscas da potência de saída nos levam a concluir que tanto o EDFA sem controle de ganho, quanto um EDFA com controle de ganho com uma dinâmica de controle lenta (ciclo de controle maior que 1 mili-segundo), não podem devem ser utilizados para amplificação em redes ópticas reconfiguráveis devido à ausência de supressão dos transientes.

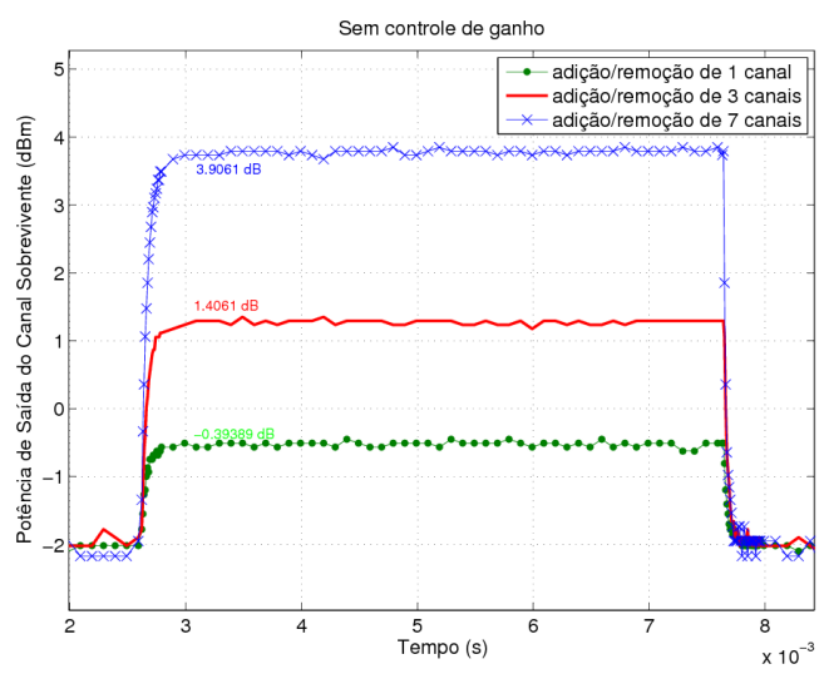

Fig. 5 - Gráfico de magnitude $(\mathrm{dBm})$ versus Tempo (s) para o canal sobrevivente no EDFA sem AGC.

A Fig. 6 ilustra o comportamento temporal para o sinal de saída do canal sobrevivente no EDFA com o controle de ganho desenvolvido. As condições para obtenção da Fig 6 são as mesmas da Fig 5 (-20 dBm por canal de entrada com potências de saída totais de $0 \mathrm{dBm}$ para 1 canal na entrada, 3 $\mathrm{dBm}$ para 2 canais na entrada, $6 \mathrm{dBm}$ para 4 canais na entrada e $9 \mathrm{dBm}$ para 8 canais na entrada). São ilustradas na Fig. 6 as situações de adição/remoção de 1 canal (variação de $3 \mathrm{~dB}$ na potência de entrada), 3 canais $(6 \mathrm{~dB})$ e 7 canais $(9$ $\mathrm{dB})$, com ganho alvo ajustado para $20 \mathrm{~dB}$. Os picos (overshoots/undershoots) dos transientes de potência para o EDFA com o controle de ganho desenvolvido foram respectivamente $0,30 \mathrm{~dB}$ (adição/remoção de 1 canal), 0,57
$\mathrm{dB}$ (adição/remoção de 3 canais) e $1 \mathrm{~dB}$ (adição/remoção de 7 canais), demonstrando claramente a eficiência da supressão de transientes utilizando a técnica de controle desenvolvida.

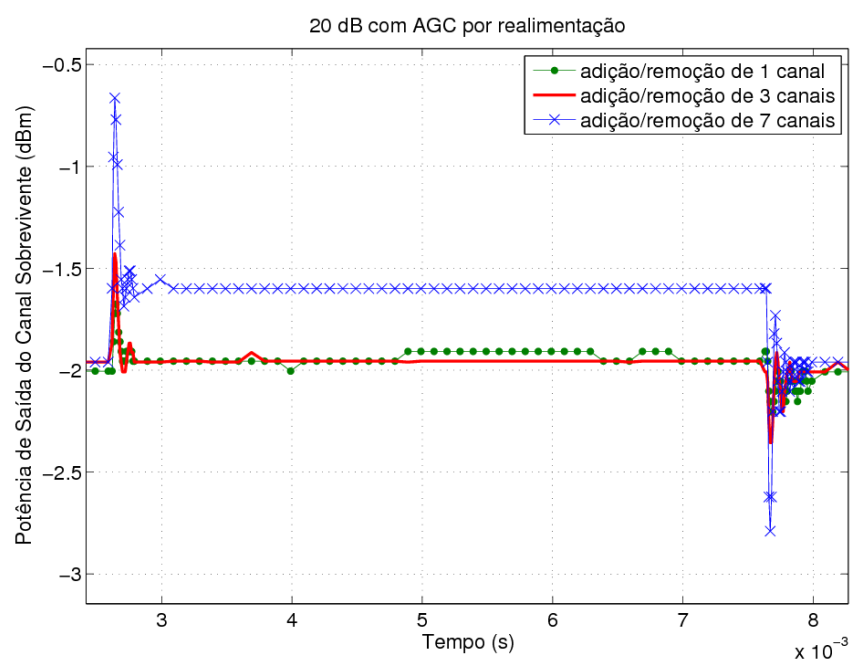

Fig. 6 - Gráfico da magnitude $(\mathrm{dBm})$ versus Tempo (s) para o canal sobrevivente no EDFA para o AGC com supressão de transientes $(\mathrm{G}=20 \mathrm{~dB})$.

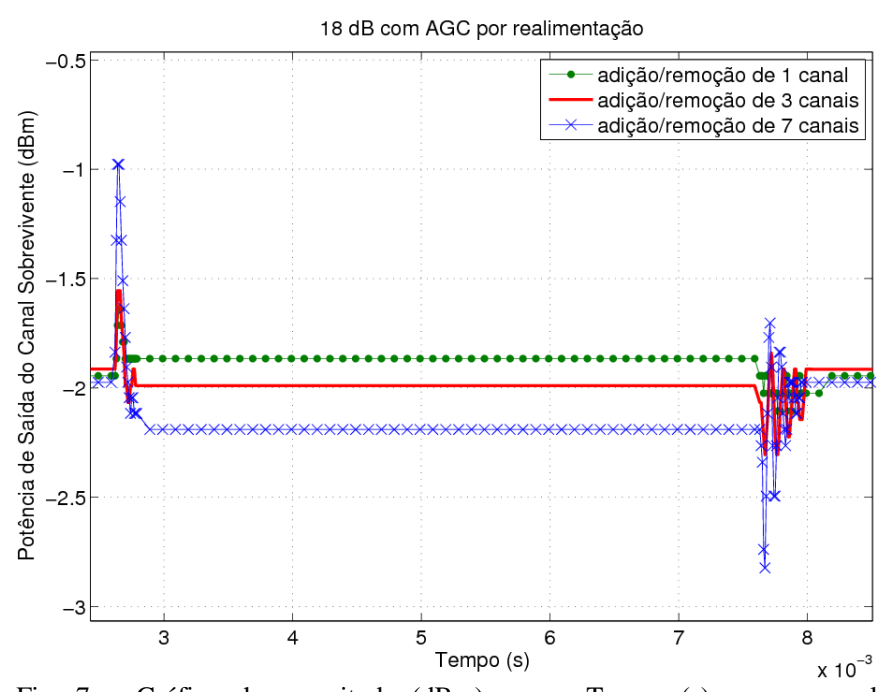

Fig. 7 - Gráfico da magnitude $(\mathrm{dBm})$ versus Tempo (s) para o canal sobrevivente no EDFA para o AGC com supressão de transientes $(\mathrm{G}=18 \mathrm{~dB})$.

Na Fig. 7, avaliamos o comportamento temporal para o sinal de saída do canal sobrevivente dado um ganho alvo ajustado para $18 \mathrm{~dB}$. Para obtenção da Fig 7 utilizamos um nível de potência de entrada de $-18 \mathrm{dBm}$ por canal, de forma que devido ao ganho de $18 \mathrm{~dB}$ ajustado, obtemos as mesmas potências de saída totais que obtemos para Fig 6. Verifica-se que o comportamento transiente do EDFA com o controle de ganho para as mesmas situações anteriores (adição/remoção de 1 canal, 3 canais e 7 canais respectivamente), apresentaram níveis máximos de overshoots/undershoots de potência de $0,33 \mathrm{~dB}, 0,46 \mathrm{~dB}$ e $1,02 \mathrm{~dB}$, respectivamente. Desta forma é demonstrado o efeito da supressão de transiente obtida pela técnica de controle desenvolvida para diferentes ganhos alvo ajustados. Quando comparado ao caso sem controle de ganho (Fig. 5), a variação da potência de saída é reduzida significativamente gerando apenas overshoots de aproximadamente $1 \mathrm{~dB}$ no pior caso analisado. 
Para o caso da adição/remoção de 7 canais $(9$ dB de variação na potência de entrada), a Fig. 8 ilustra a relação entre o EDFA sem controle de ganho e com o controle de ganho desenvolvido para o ganho ajustado de $18 \mathrm{~dB}$. Através da Fig 8 temos como comparar visualmente o nível de supressão de transientes alcançado pelo EDFA com o AGC com supressão de transientes desenvolvido frente ao EDFA sem AGC.

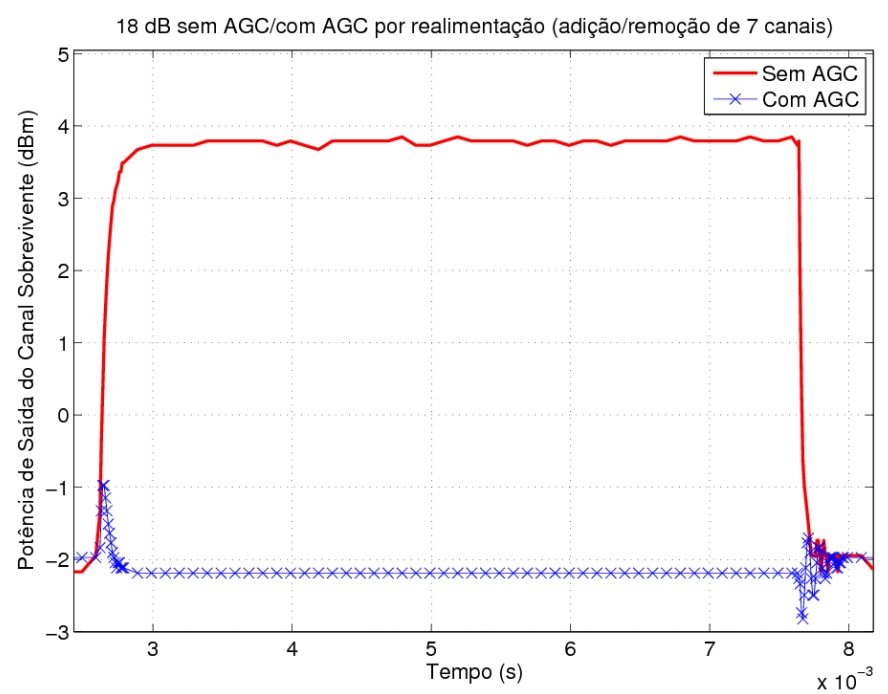

Fig. 8 - Gráfico da magnitude $(\mathrm{dBm})$ versus Tempo (s) para o canal sobrevivente no EDFA sem AGC e com AGC com supressão de transientes.

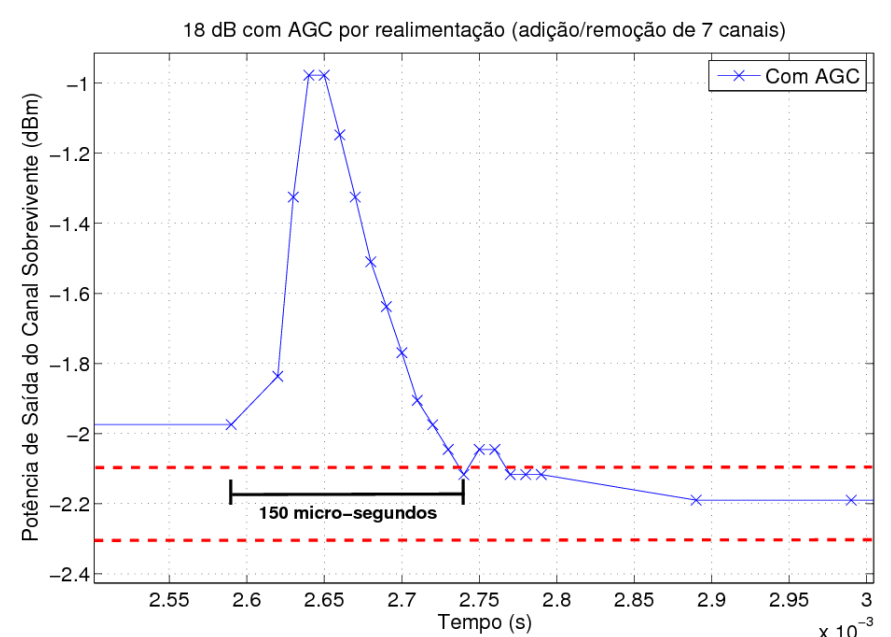

Fig. 9 - Gráfico da magnitude $(\mathrm{dBm})$ versus Tempo (s) para o canal sobrevivente no EDFA para o AGC com supressão de transientes.

A Fig 9 ilustra com maiores detalhes o comportamento da potência de saída do canal sobrevivente no momento da remoção de $9 \mathrm{~dB}$ na potência de entrada para o EDFA com controle de ganho com supressão de transientes desenvolvido. Considerando o limite superior do erro de +/$5 \%$ em torno do valor de regime permanente (ou seja, $95 \%$ do controle de ganho realizado), obtêm-se um settling time de aproximadamente um de $150 \mu \mathrm{s}$, que se encontra dentro do limite de tempo do controle especificado em [9]-[10].

Assim, verificamos que os objetivos relativos à supressão de transiente e ao tempo de resposta foram alcançados, dado. Dessa maneira, conclui-se que a técnica de controle de ganho desenvolvida atende aos requisitos temporais exigidos, e que, com os tempos de resposta e picos de potências transientes obtidos, esta técnica desenvolvida posiciona-se no estado da arte do controle automático de ganho digital aplicado a EDFAs.

\section{B. Análise Sistêmica Qualitativa (Diagrama de Olho)}

Para avaliação do desempenho sistêmico do EDFA desenvolvido foi utilizado o arranjo experimental ilustrado na Fig. 4. O desempenho sistêmico do EDFA foi realizado através da análise dos resultados do diagrama de olho para o canal sobrevivente modulado a $10 \mathrm{~Gb} / \mathrm{s}$, para o EDFA sem e com controle de ganho para fins de comparação relativos ao desempenho do EDFA com a técnica proposta em um cenário de rede óptica reconfigurável.

Para as condições de ganho ajustadas no EDFA (18 dB e 20 dB) a entrada do EDFA foi submetida a 100 adições/remoções por segundo durante 10 segundos (1000 amostras), realizando os eventos de remoção e inserção de 1 (3 dB de variação da potência de entrada), $3(6 \mathrm{~dB})$ e $7(9 \mathrm{~dB})$ canais na entrada do EDFA. Para obtenção dos diagramas de olho apresentados nas Figs. 10 e 11, a potência de entrada por canal foi de $-20 \mathrm{dBm}$ para o ganho ajustado de $20 \mathrm{~dB},-18$ $\mathrm{dBm}$ para o ganho ajustado de $18 \mathrm{~dB}$, e as potências de saída totais foram iguais as obtidas nas Figs 6 e 7

As Figs. 10 e 11 ilustram os diagramas de olhos obtidos no pior caso sob análise (adição/remoção de $9 \mathrm{~dB}$ da potência de entrada - sete canais) para os ganhos ajustados.

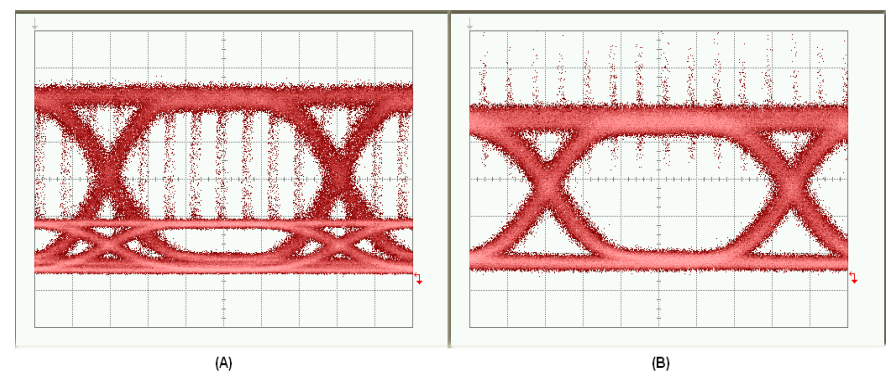

Fig. 10 - Diagramas de olho para ganho de $20 \mathrm{~dB}$. (A) canal sobrevivente sem controle de ganho (395 $\mu \mathrm{W} /$ div), (B) canal sobrevivente com controle de ganho $(184 \mu \mathrm{W} / \mathrm{div})$.
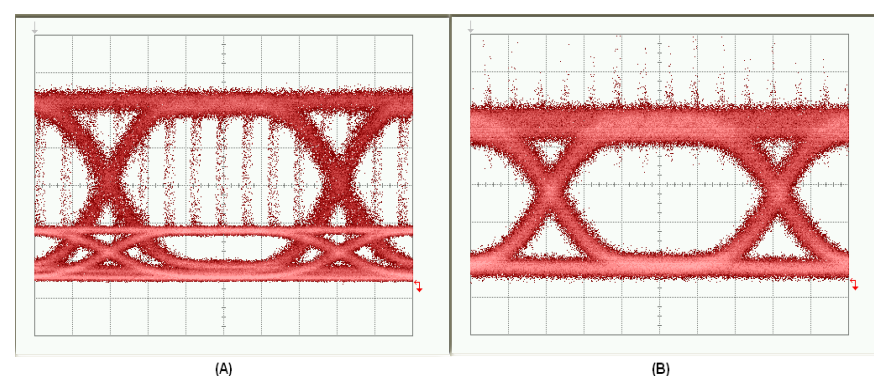

Fig. 11 - Diagramas de olho para ganho de $18 \mathrm{~dB}$. (A) canal sobrevivente sem controle de ganho ( $395 \mu \mathrm{W} / \mathrm{div})$, (B) canal sobrevivente com controle de ganho $(111 \mu \mathrm{W} / \mathrm{div})$.

As Figs. 10 (A) e 11 (A) ilustram os diagramas de olho para o ganho ajustado em $20 \mathrm{~dB}$ e $18 \mathrm{~dB}$, respectivamente, para o EDFA sem controle de ganho. Já as Figs. 10 (B) e 11 (B) ilustram o diagrama de olho para o EDFA utilizando a 
técnica de controle de ganho com supressão de transiente proposta neste trabalho. Nas Figs. 10 e 11 (A), verifica-se um olho completamente penalizado, que não apresenta nenhuma abertura capaz de permitir a definição de um limiar de decisão. Nas Figs. 10 (B) e 11 (B), verifica-se um diagrama de olho onde claramente pode-se determinar um limiar de decisão para o receptor, visualizamos também alguns pontos pouco acima e pouco abaixo do nível de bit " 1 ". Estes pontos são adquiridos durante o transiente de potência de $150 \mu \mathrm{s}$, porém mesmo assim conseguimos determinar um limiar de decisão para o receptor, removendo praticamente todos os efeitos causados pela inserção e remoção de canais. Assim, através da análise qualitativa baseada nos diagramas de olho, verificam-se os benefícios sistêmicos associados ao EDFA com controle automático eletrônico de ganho desenvolvido, o qualificando para uso em redes ópticas WDM reconfiguráveis.

\section{CONCLUSÃO}

Neste trabalho foi demonstrado experimentalmente o desenvolvimento de uma técnica de controle de ganho ultrarápida capaz de habilitar o uso de EDFAs em redes WDM reconfiguráveis. Foi obtido um controle eletrônico com ciclo de atuação de $9 \mu \mathrm{s}$, com settling time de máximo de $150 \mu \mathrm{s}$, e máximo undershoot/overshoot de $1,02 \mathrm{~dB}$, o que posiciona esta técnica no estado da arte de amplificação óptica em termos de supressão dos efeitos transientes nos EDFAs. Foi verificado o impacto sistêmico, incluindo o transiente, para sistemas com taxa de $10 \mathrm{Gbit} / \mathrm{s}$ por canal, onde se comprovou a aplicabilidade desta técnica de controle de ganho para EDFAs, mesmo frente aos eventos de add/drop de canais nas redes ópticas reconfiguráveis.

\section{REFERÊNCIAS}

[1] Ding, M., and Pavel, L., "Gain Scheduling Control Design of an Erbium Doped Fibre Amplifier by Pump Compensation", Proc. IEEE Conference on Control Applications, pp.510-516, Aug. 2005

[2] X. Feng et al., "A simple control algorithm for wide-band channel-power. clamped EDFA,” Opt. Commun., vol. 213, pp. 285-292, Dec. 1, 2002.

[3] J. L. Zyskind, et. al, "Fast-link control protection of surviving channels in multiwavelength optical networks," IEEE Photon. Technol.Lett., vol. 9, pp. 1667-1669, Dec. 1997.

[4] B. Clesca, V. Havard, S. Gauchard, V. Rodrigues, E. Lantonien, D. Cravec, and F. X. Ollivier, "Upper limit and control scheme for power per channel in optically-amplified WDM systems," in Proc. ECOC's96, vol. 9, 1996, pp. 333-336.

[5] L. Pavel, "Control design for transient power and spectral control in optical communication networks," in Proc. IEEE Conference on Control Applications, vol. 1, June 2003, pp. $415-422$.

[6] L. Tancevski, L. A. Rusch, and A. Bononi, "Gain control in EDFA's by pump compensation" IEEE Photon. Technol. Lett., vol. 10, no. 9, pp. 13131315, Sept. 1998.

[7] S. Y. Park, et. al, "Dynamic gain and output power control in gainflattened erbium-doped fiber amplifier," IEEE Photon. Technol. Lett., vol. 10, no. 6, pp. 787-789, June 1998.

[8] K. Motoshima, N. Suzuki, and K. Shimizu, "A channel-number insensitive erbium-doped fiber amplifier with automatic gain and Power regulation function," J. Lightwave Technol., vol. 19, no. 11, pp. 1759-1767, Nov. 2001.

[9] A. Srivastava, "What is Next for Optical Amplifiers?" Oneterabit presentation, 2006. http://tc86.iec.ch/news/pdf_2007/Srivastava.pdf 30/07/2009.
[10] A. K. Srivastava, Y. Sun, J. L. Zyskind, J.W.Sulhoff, "EDFA Transient Response to Channel Loss in WDM Transmission System", IEEE Photon. Technol. Lett., vol. 9, no. 3, 386-388, 1997.

[11] J. C. R. F. de Oliveira,"Amplificadores Ópticos com Controle Automático de Ganho para aplicações em Redes Ópticas Reconfiguráveis", Campinas State University, Brazil, Phd thesis, 2007.

[12] E. Desurvire, "Analysis of transient gain saturation and recovery in erbium-doped fiber amplifiers", IEEE Photon. Technol. Lett., vol. 1, no. 8, pp. 196-199, 1989.

[13] C. R. Giles e E. Desurvire, "Transient gain and crosstalk in erbiumdoped fibre amplifiers", Op. Let., Vol. 14, no. 16, pp. 880-882, 1989.

[14] Y. Sun e A. K. Srivastava, "Dynamic effects in optically amplified networks", em Proc. Conference on Optical Amplifiers and their Applications, OAA '97, pp. 44-47, 1997. 